\title{
METHOD TO CORRELATE CFD DISCRIMINATOR LEVEL AND ENERGY DEPOSITION BY NEUTRONS AND PHOTONS IN A FAST PLASTIC SCINTILLATING DETECTOR
}

\author{
Lisa G. Chiang \\ Richard B. Oberer \\ Oak Ridge National Laboratory \\ P.O. Box 2008 \\ Oak Ridge, TN 37831-6004 USA
Sara A. Pozzi ${ }^{*}$
*Department of Nuclear Engineering
Polytechnic of Milan Italy

June 26, 2000

\author{
Prepared by \\ OAK RIDGE NATIONAL LABORATORY \\ Oak Ridge, Tennessee 37831-6004 \\ managed by \\ UT-BATTELLE, LLC \\ for the \\ U. S. DEPARTMENT OF ENERGY \\ under contract DE-AC05-00OR22725
}




\section{CONTENTS}

Page

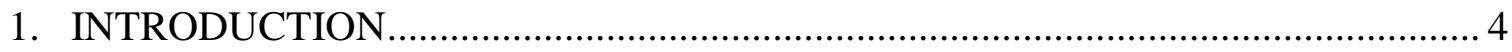

1.1 DETECTOR NEUTRON COUNTING EFFICIENCY .................................... 4

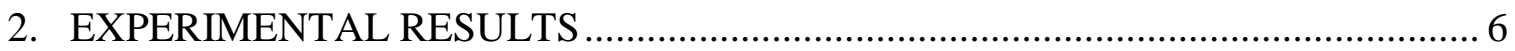

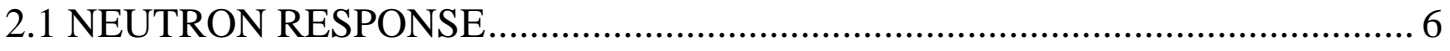

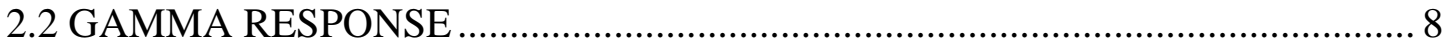

3. COMPARISON OF NEUTRON AND GAMMA RESPONSE .............................. 10

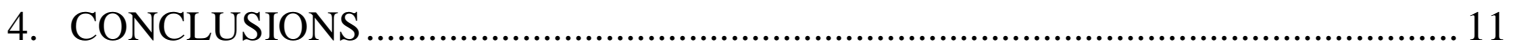




\section{LIST OF FIGURES}

Page

Fig. 2.1 Detector efficiency for neutrons for three different CFD discriminator levels: 25, 45 and $75 \mathrm{mV}$. Dashed lines are fits of experimental data points to Equation (3).

Fig. 2.2 Neutron energy threshold as a function of CFD discriminator level:

experimental points fitted with $\mathrm{E}_{\mathrm{th}}=0.017 \mathrm{~V}_{\mathrm{CFD}}+0.47$

Fig. 2.3. Detector response to gamma rays shown for various gamma sources.

Fig. 2.4 Derivative of detector gamma response: experimental data for ${ }^{137} \mathrm{Cs}$ and

${ }^{133} \mathrm{Ba}$. Maximum value is shown with a circle, half height of this maximum is shown with a square and selected Compton edge value is shown with a triangle.

Fig. 3.1 Response data for gamma rays and neutrons as a function of the incident

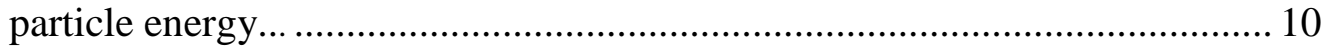

Fig. 3.2 Neutron to gamma ray energy ratio as a function of CFD discriminator level $(\mathrm{mV})$. 


\begin{abstract}
This paper presents an experimental approach to determine the neutron and gamma ray thresholds in fast, organic scintillating detectors. Neutron threshold energy is determined using a time-of-flight technique, while gamma ray threshold is inferred by locating the Compton edge for various reference gamma sources. These energy thresholds are then related to the Constant Fraction Discriminator (CFD) levels used in these experiments.
\end{abstract}

\title{
1. INTRODUCTION
}

In fast correlation measurements performed with Nuclear Materials Identification System $^{1}$ (NMIS, formerly NWIS), the output of the plastic scintillating detector is input to a constant fraction discriminator (CFD) which produces a fast NIM signal when a detector pulse exceeds the CFD discriminator level. This discriminator level can be set from $20 \mathrm{mV}$ to $1000 \mathrm{mV}$. In the MCNP-DSP ${ }^{2}$ simulation of the experiment, the neutron and gamma ray thresholds are independently selected in terms of energy deposited in a detector rather than in terms of the detector output signal. It is therefore important to correlate the CFD discriminator level to the theoretical gamma and neutron energy thresholds. The CFD discriminator level selects a neutron to gamma ray threshold energy ratio.

A problem arises because the amplitude of the output signal from a detector depends not only on the energy deposited in the detector by a radiation but also on the mechanism of the energy deposition. For a given amount of energy deposited, gamma rays produce more fluorescent energy than neutrons, resulting in larger detector output amplitudes for gamma rays than neutrons.

The reason for the difference in detector response is that gamma rays deposit energy in the detector primarily through Compton scattering interactions with the electrons in the detector whereas neutrons deposit energy primarily through elastic scattering with the nuclei of hydrogen atoms in the detector. Therefore, gamma radiation deposits energy in the detector through electrons while neutrons deposit energy through protons. For a given energy, the stopping power of a proton is greater than that of an electron. Because of this difference, protons deposit energy more densely than electrons. The assumption is that the higher density of the energy deposition leads to quenching due to damaged molecules in the detector. ${ }^{3}$

\subsection{DETECTOR NEUTRON COUNTING EFFICIENCY}

The neutron counting efficiency of the detector versus CFD discriminator level was used to calibrate the detector response per neutron energy deposited. ${ }^{3}$ The detection efficiency goes to zero at the neutron energy corresponding to the threshold voltage. This zero crossing energy was determined by fitting the experimental data to the following theoretical efficiency curve: 


$$
\varepsilon\left(E_{n}\right)=\frac{k}{E_{n}}\left(1-\frac{E_{t h}}{E_{n}}\right)
$$

where $E_{t h}$ is the neutron threshold energy.

The detector efficiency as a function of neutron energy was measured by a time of flight method. The covariance between an instrumented ${ }^{252} \mathrm{Cf}$ source and the detector represents the time after fission that a gamma or neutron is detected. At one meter the gamma rays from fission arrive at the detector at $3.3 \mathrm{nsec}$. The neutron arrival times can be represented by the Maxwellian time distribution. In terms of energy, a ${ }^{252} \mathrm{Cf}$ fission spectrum is approximated by the following Maxwellian distribution:

$$
f\left(E_{n}\right) d E_{n}=\frac{2}{\sqrt{\pi}} \frac{\sqrt{E_{n}}}{T^{2 / 3}} e^{-\frac{E}{T}} d E_{n}
$$

where $T$ is the nuclear temperature taken to be $1.42 \mathrm{MeV}$ and $E_{n}$ is the neutron energy. The number of neutrons impinging on the detector in the energy bin from $E_{1}=\frac{1}{2} m\left(\frac{d}{t_{1}}\right)^{2}$ to $E_{2}=\frac{1}{2} m\left(\frac{d}{t_{2}}\right)^{2}$ is $N\left(E_{n}\right)=S \bar{v} g{ }_{E_{1}}^{E_{2}} f\left(E_{n}\right) d E_{n}$ where $S$ is the detected fission rate from the instrumented ${ }^{252} \mathrm{Cf}$ source, $\bar{v}$ is the average number of neutron emitted per fission and $\mathrm{g}$ is a geometry factor.

The detector efficiency is then $\varepsilon\left(E_{n}\right)=\frac{C\left(E_{n}\right)}{N\left(E_{n}\right)}$ where $C\left(E_{n}\right)$ is the number of counts recorded by the detector within the delay interval $t_{1}$ and $t_{2}$ from the covariance. There is a slight ambiguity in the energy $E_{n}$ because a range of energies is involved. Usually this ambiguity is resolved by letting $E_{n}=\frac{E_{1}+E_{2}}{2}$ which is valid when the energy bins are small. 


\section{EXPERIMENTAL RESULTS}

The detector used in the measurements is a commercially available plastic scintillator sensitive to fast neutrons and gamma rays. The crystal, BC 420 (Bicron) has nominal dimensions of 3"x 3" x 4". The high voltage setting for the photomultiplier tube (PMT) of the detector is selected and kept constant throughout the experiment. The signal from the anode of the PMT base is sent to a CFD, ORTEC Model 935, which eliminates pulses having amplitude lower than a given discriminator level.

\subsection{NEUTRON RESPONSE}

Time-of-flight measurements were performed with varying CFD discriminator levels. The instrumented ${ }^{252} \mathrm{Cf}$ source was placed at a distance of $1 \mathrm{~m}$ from the detector. Efficiency data was acquired for CFD discriminator levels varying from 25 to $75 \mathrm{mV}$. Three of these efficiency curves are shown in Figure 2.1.

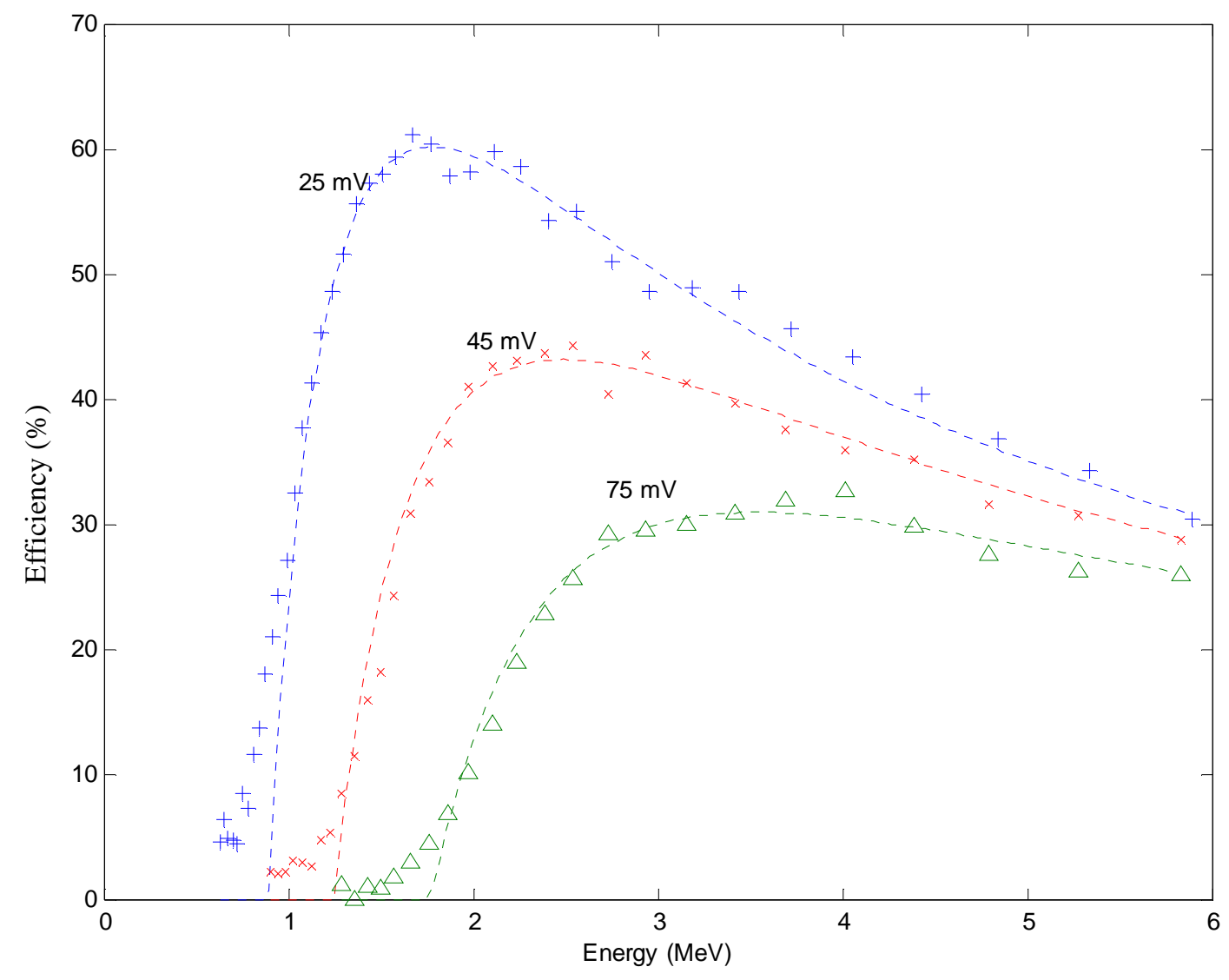

Fig. 2.1. Detector efficiency for neutrons for three different CFD discriminator levels: 25,45 and $75 \mathrm{mV}$. Dashed lines are fits of experimental data points to Equation (2). 
The threshold energy $E_{t h}$ was selected using the zero cross-over point of the theoretical fit given in Equation (2). Figure 2.2 shows $E_{t h}$ as a function of the CFD discriminator level. Also shown is a linear fit to the experimental values.

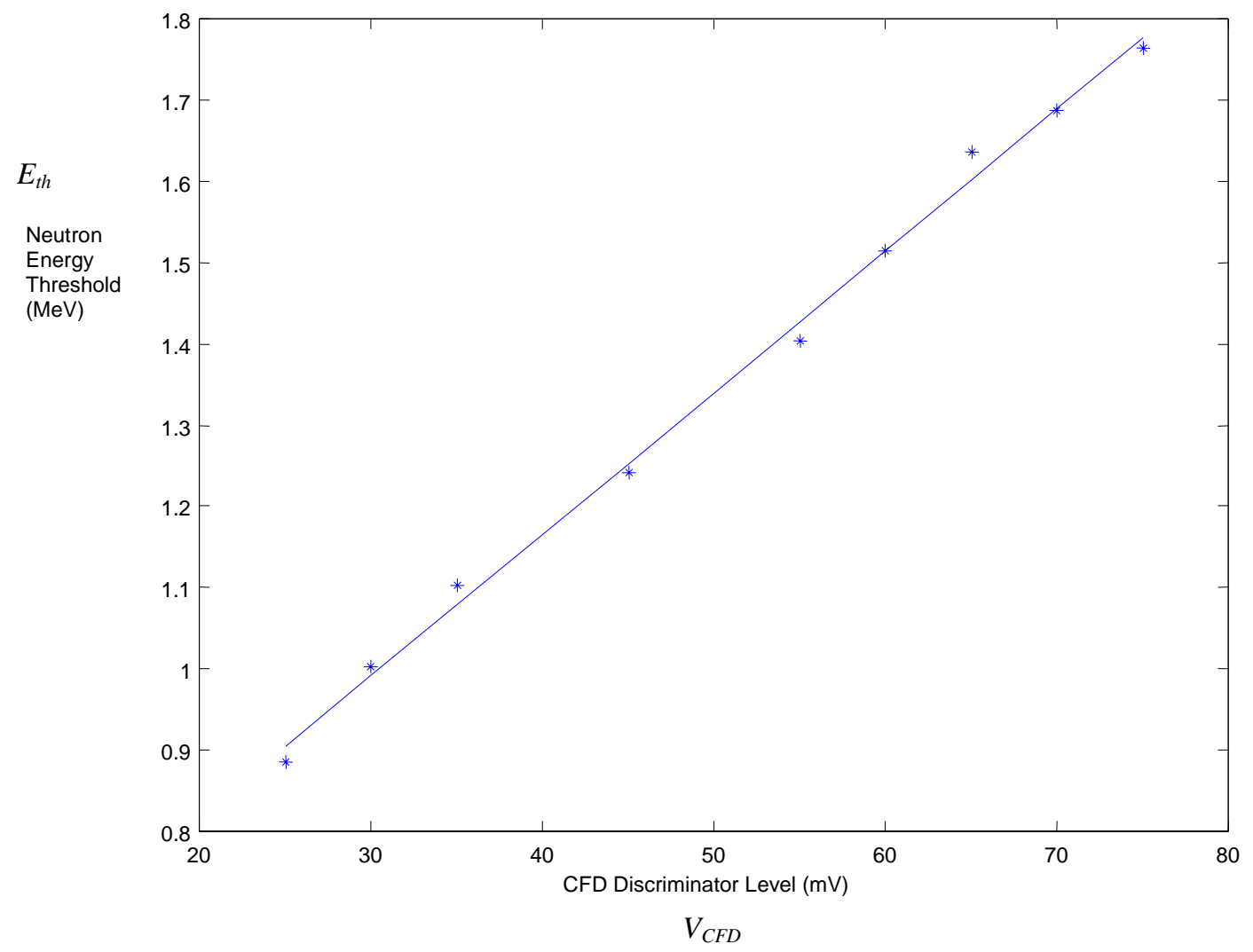

Fig. 2.2. Neutron energy threshold as a function of CFD discriminator level $\left(V_{\text {CFD }}\right)$ : experimental points fitted with $E_{\text {th }}=0.017 V_{C F D}+0.47$. 


\subsection{GAMMA RESPONSE}

In this setup the output of the CFD was counted and accumulated over a 10 second period. The counter records integrated counts in the Compton plateau above the gamma threshold. In order to correlate the gamma threshold to the CFD discriminator level, the measurement was repeated for different gamma sources ${ }^{4}$ and with varying CFD discriminator levels. The results are shown in Figure 2.3 for various gamma sources: ${ }^{137} \mathrm{Cs},{ }^{22} \mathrm{Na},{ }^{60} \mathrm{Co},{ }^{54} \mathrm{Mn}$, and ${ }^{133} \mathrm{Ba}$. The location of the Compton edge was determined by taking the derivative of the integrated counts.

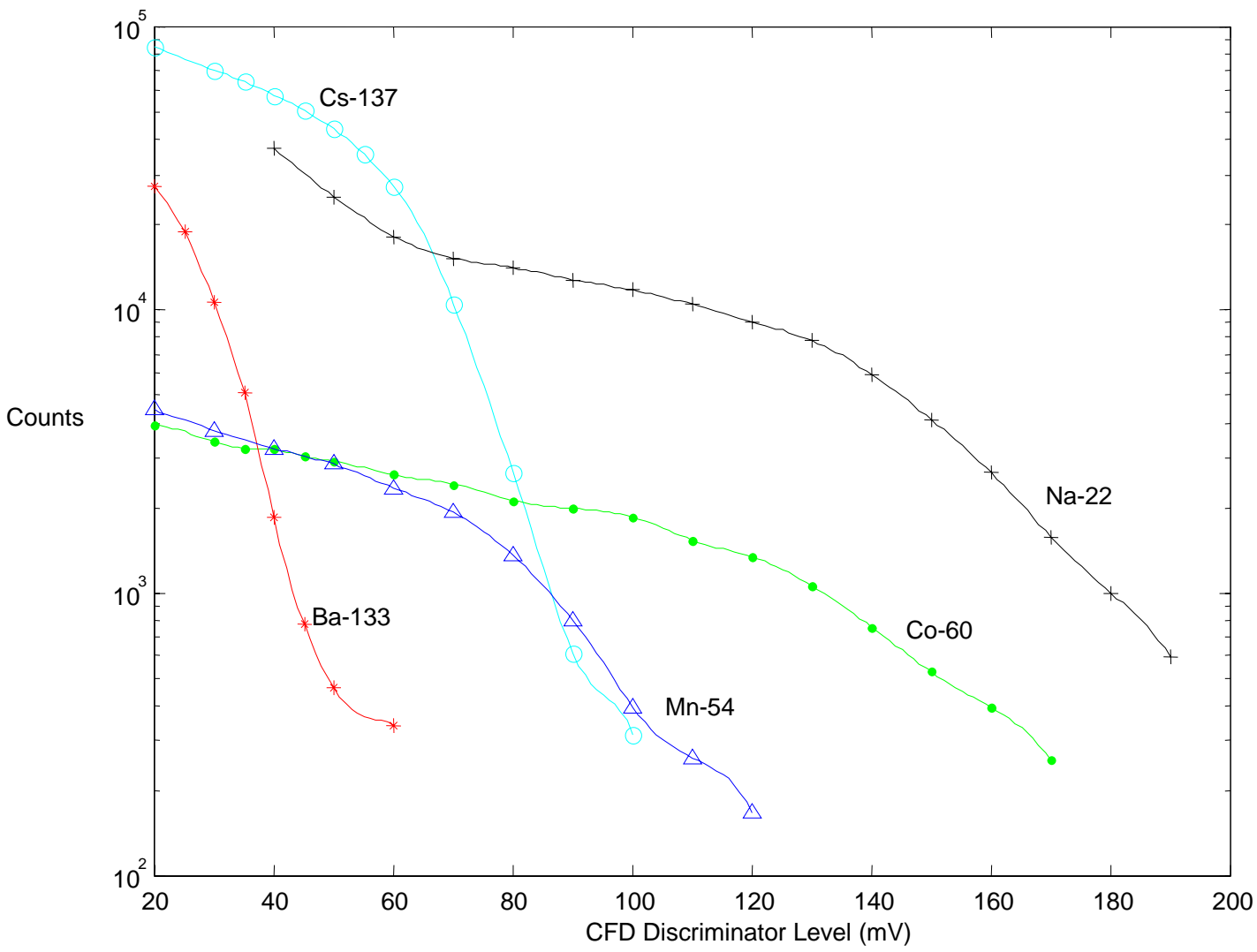

Fig. 2.3. Detector response to gamma rays shown for various gamma sources.

Previous studies ${ }^{5}$ have shown that the position of the Compton edge depends upon detector resolution. In our application, the energy corresponding to the midpoint between the Compton peak and half its height was chosen as the Compton edge.

The derivative of the detector response is shown in Figure 2.4 for ${ }^{137} \mathrm{Cs}$ and ${ }^{133} \mathrm{Ba}$, along with the position of the Compton edge. The gamma energies of the sources are well known (Table 1) and can be used to find a relationship between gamma ray energy $(\mathrm{MeV})$ and $\mathrm{CFD}$ threshold $(\mathrm{mV})$. 


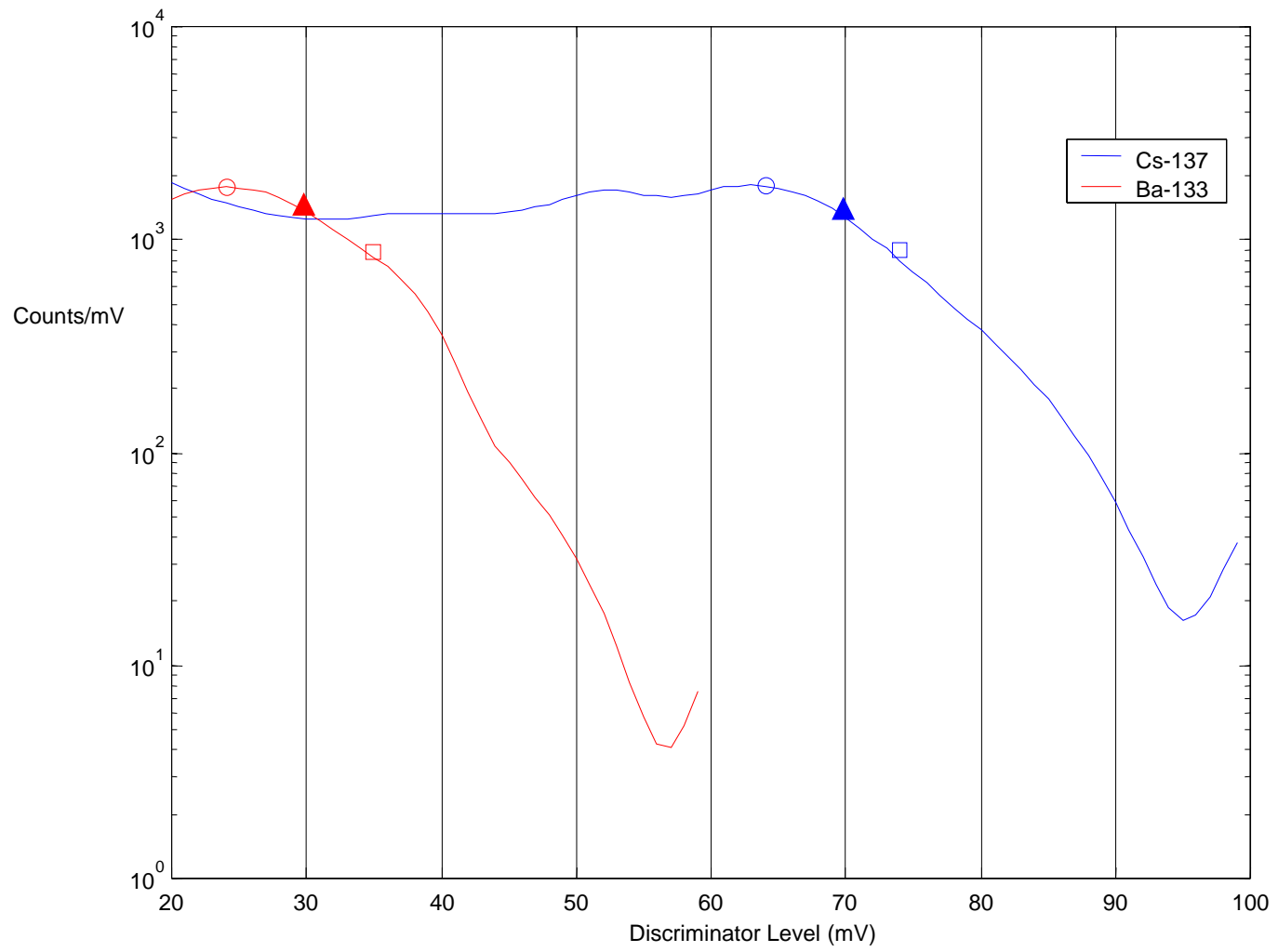

Fig. 2.4. Derivative of detector gamma response: experimental data for ${ }^{137} \mathrm{Cs}$ and ${ }^{133} \mathrm{Ba}$. Maximum value is shown with a circle, half height of this maximum is shown with a square and selected Compton edge value is shown with a triangle.

Table 1. Data of gamma sources and detector response

\begin{tabular}{|l|c|c|c|c|}
\hline \multirow{3}{*}{ Isotope } & $\begin{array}{c}\text { Gamma } \\
\text { Energy } \\
(\mathrm{MeV})\end{array}$ & $\begin{array}{c}\text { Intensity } \\
\%\end{array}$ & $\begin{array}{c}\text { Compton Edge } \\
(\mathrm{MeV})\end{array}$ & $\begin{array}{c}\text { Discriminator level } \\
\text { corresponding to } \\
\text { Compton edge } \\
(\mathrm{mV})\end{array}$ \\
\hline \multirow{3}{*}{ Ba-133 } & 0.3031 & 18.33 & 0.164 & \multirow{2}{*}{30.5} \\
\cline { 2 - 4 } & 0.356 & 62.05 & 0.207 & 70 \\
\cline { 2 - 4 } Cs-137 & 0.384 & 8.94 & 0.230 & 89.5 \\
\hline Mn-54 & 0.662 & 85.1 & 0.477 & 148 \\
\hline Co-60 & 0.835 & 99.98 & 0.639 & 134 \\
\cline { 2 - 4 } & 1.173 & 99.97 & 0.963 & 1.118 \\
\hline Na-22 & 1.333 & 99.99 & 1.154 & \\
\hline
\end{tabular}




\section{COMPARISON OF NEUTRON AND GAMMA RESPONSE}

The detector response for neutrons and gamma rays can be compared. Figure 3.1 shows the scintillator response as a function of the energy of the incident particle. Linear regressions showed the photon data to be linear with $\mathrm{r}^{2}$ of 0.991 , and $\mathrm{r}^{2}$ of 0.996 for the neutron data. Figure 3.2 shows the ratio of neutron-to-gamma ray energy. This ratio varies from 3.2 to 4.6 , in good agreement with results found in literature ${ }^{3,4}$. The neutronto-gamma ray energy ratio decreases with increasing CFD discriminator level.

CFD

Discriminator Level (mV)

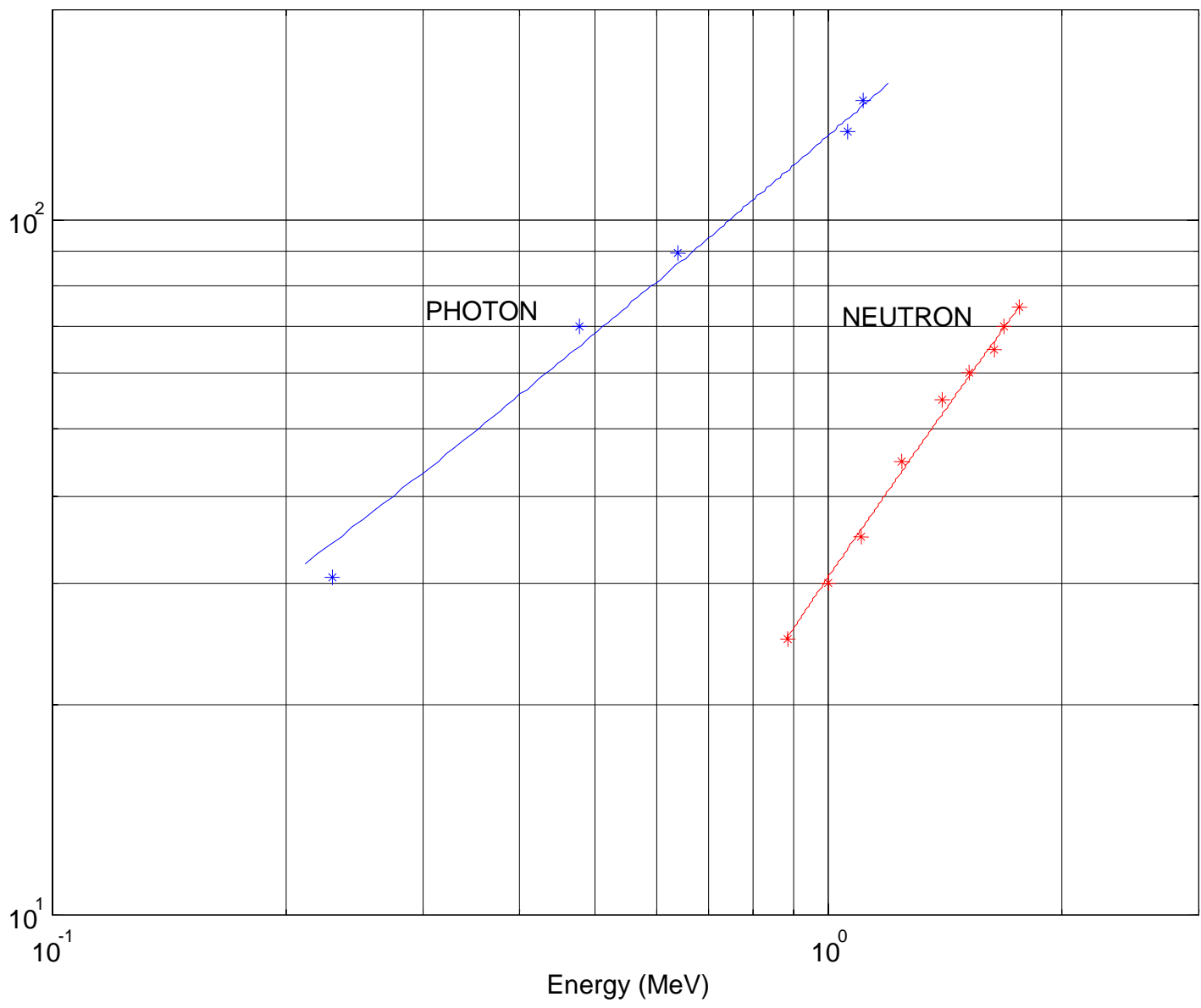

Fig. 3.1. Response data for gamma rays and neutrons as a function of the incident particle energy. 


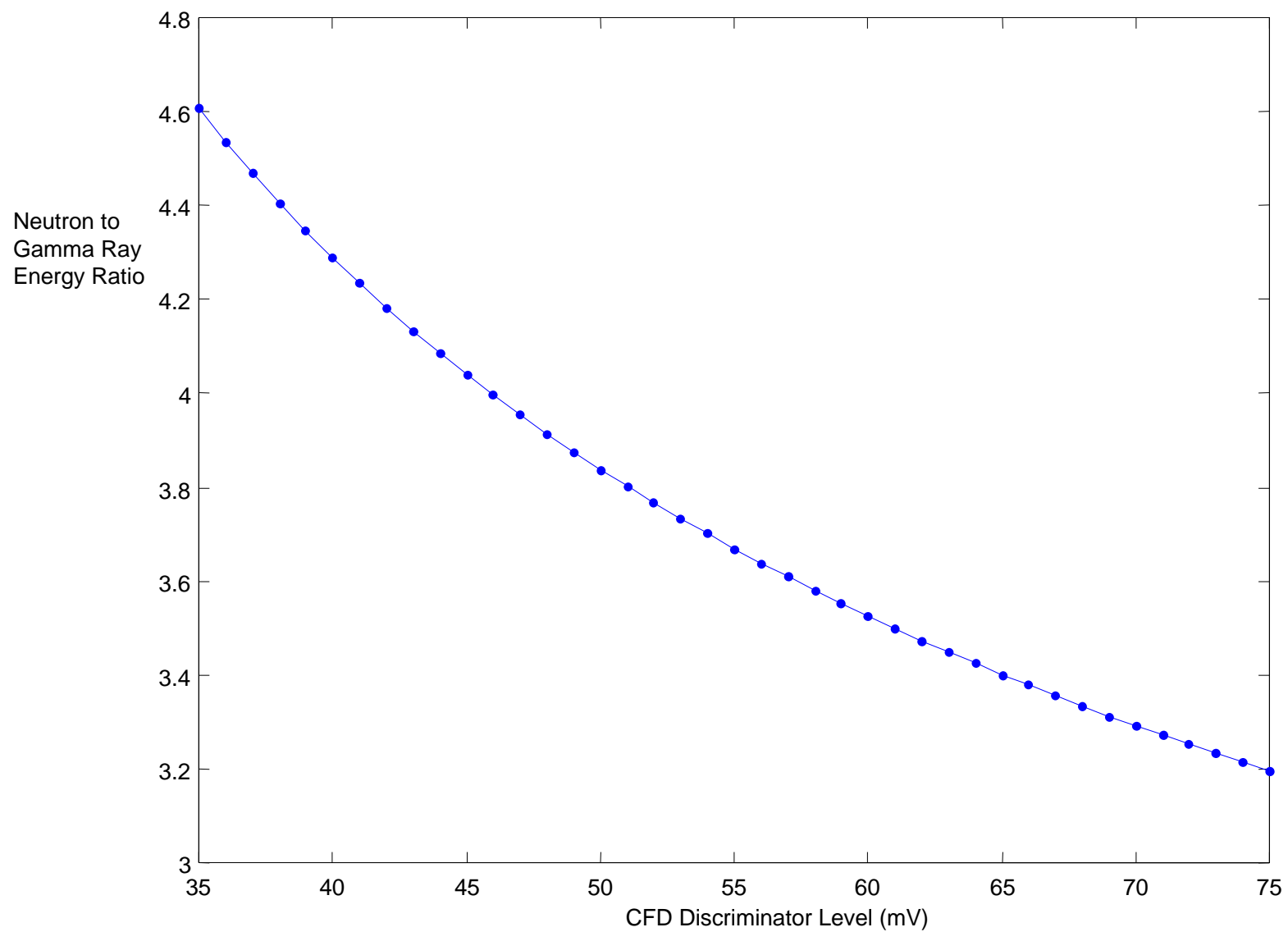

Fig. 3.2. Neutron to gamma ray energy ratio as a function of CFD discriminator level (mV).

\section{CONCLUSIONS}

In this paper we have presented a method to correlate CFD discriminator level and energy deposition by neutrons and photons in a fast plastic scintillating detector. Neutron response data has been determined using the NMIS to acquire time-of-flight signatures at varying CFD discriminator levels. A number of gamma sources have been used to determine the gamma ray response of the detector. The results are in good agreement with those found in literature, as well as those adopted in the past few years of NMIS operation. 


\section{REFERENCES}

1. J. T. Mihalczo, T. E. Valentine, and J. K. Mattingly, NWIS Methodology, Y/LB15,953, Oak Ridge Y-12 Plant (1997).

2. T. E. Valentine, MCNP-DSP Users Manual, ORNL/TM-13334, Oak Ridge National Laboratory, January 1997.

3. G. F. Knoll, Radiation Detection and Measurement, $2^{\text {nd }}$ ed., 1989, John Wiley, pp. 222, 538.

4. R. L. Craun, D. L. Smith, Analysis of Response Data for Several Organic Scintillators, Nuclear Instruments and Methods 80 (1970).

5. G. Dietze, H. Klein, Gamma-Calibration of NE 213 Counters, Nuclear Instruments and Methods 193 (1982).

6. D. F. Flynn, L. E. Glendenin, et al., Pulse Height-Energy Relations for Electrons and Alpha Particles in a Liquid Scintillator, Nuclear Instruments and Methods 27 (1964). 


\section{DISTRIBUTION}

1. L. G. Chiang

2. J. K. Mattingly

3. J. A. McEvers

4. J. T. Mihalczo

5. J. A. Mullens

6. R. B. Oberer

7. S. A. Pozzi

8. T. E. Valentine

9. J. D. White

10. M. C. Wright

11. ORNL Laboratory Records $-\mathrm{RC}$ 